\title{
Successful Management of Stress Urinary Incontinence in Women by a Contraceptive Device FemCap ${ }^{\text {tw }}$ Pilot Study
}

\author{
Alfred Shihata ${ }^{1^{\star}}$, Steven A Brody ${ }^{2}$, Julia Barrett-Mitchell ${ }^{3}$ \\ ${ }^{1}$ Scripps Institution of Medicine and Science San Diego, CA, USA \\ ${ }^{2}$ Director of Life Span Medical Institute San Diego, CA, USA \\ ${ }^{3}$ FemCap Inc., Clinical coordinator, 14058 Mira Montana Drive \\ Del Mar CA, USA
}

\author{
*Corresponding author \\ Alfred Shihata, Scripps Institution of Medicine and Science San Diego, CA, \\ USA
}

Submitted: 20 Sep 2020; Accepted: 06 Oct 2020; Published: 11 Oct 2020

\begin{abstract}
Background: Women suffer silently from stress urinary incontinence (SUI). SUI is under-reported by women and under-diagnosed and treated by doctors. Treatment with pessaries is conservative and has significant limitations. These limitations include displacement, erosion, ulceration, and urethral obstruction. (SUI) is very prevalent among women of all ages, particularly menopausal women. The first line of SUI treatment is the ring pessary; however, more pessaries of different shapes and sizes have been introduced to achieve better results.
\end{abstract}

Objectives: To provide women with a safer, more effective device to treat SUI.

Materials and Methods: The Fem Cap combines the ring and space-occupying pessary features into one device, making it much more successful. The bowl of the dome of the FemCap covers the cervix and prevents it from prolapsing. The rim fits snugly into the vaginal fornices that support the bladder neck. The brim flares outward, pushing against the cystocele and the urethrocele anteriorly to restore the urethra and the bladder's anatomy. Forty-one women who had had significant SUI were recruited and asked to compare their experience for one week before using the FemCap and two weeks later. The FemCap was self-inserted and removed by the participants.

Results: Thirty-four women out of 41 were completely dry after two weeks of using the FemCap, while four women were partially dry, and three women did not notice any change. The participants reported no side effects, and pelvic examinations did not show any erosion or ulceration of the vagina.

Conclusion: The FemCap is safe and effective in restoring the bladder and urethra's anatomy, which could make it ideal for the treatment and prevention of mild to moderate SUI. More studies are warranted to prove the utility of the FemCap further to manage SUI.

Keywords: FemCap, FemContinence, Stress Urinary Incontinence

\section{Introduction}

Stress urinary incontinence (SUI) is prevalent among women of all ages, particularly menopausal women. SUI is under-reported by women as well as under-diagnosed and treated by doctors. A woman using the FemCap for contraception reported that she had suffered from stress urinary incontinence, but her SUI subsided when using the FemCap (Figure 1,2,3) [1-8]. Thankfully, she shared her experience, which led me to investigate a new usage for the FemCap as an SUI pessary called FemContinence. The first line of therapy of SUI is pelvic floor muscle Kegel exercises and vaginal pessaries. (Figure 4). The most popular is the ring pessary (Figure 5); however, a variety of shapes and sizes (Figure 6,7) are becoming available to achieve better results $[9,10]$.

It should be noted that pessaries are NOT a cure but are safe and effective in treating mild to moderate SUI. 


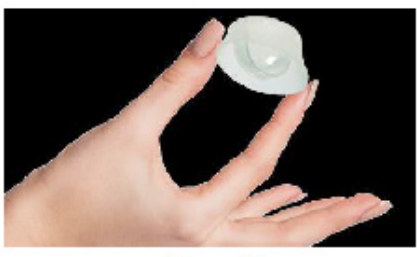

Figure 1

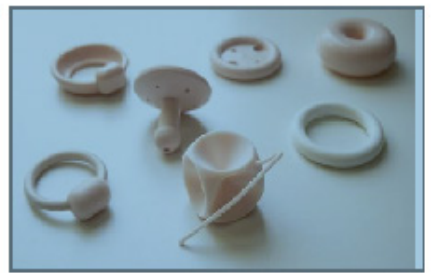

Figure 4

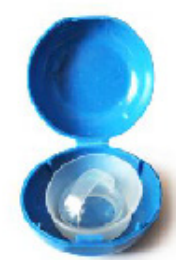

Figure 2

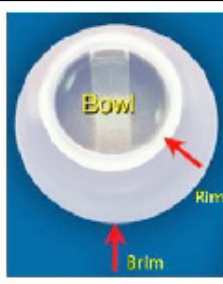

Figure 3

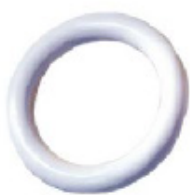

Figure 5

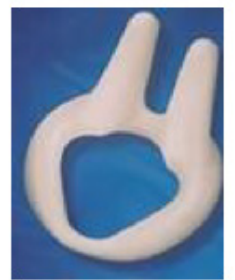

Figure 6
How uresta ${ }^{8}$ works
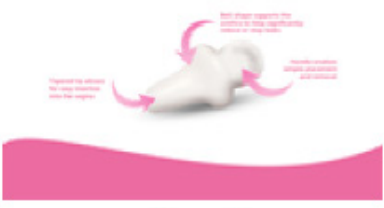

Figure 7

Pessaries are conservative alternatives to surgical repair, which is done with a sling for pelvic organ prolapse (POP) and SUI and (Figure 8).

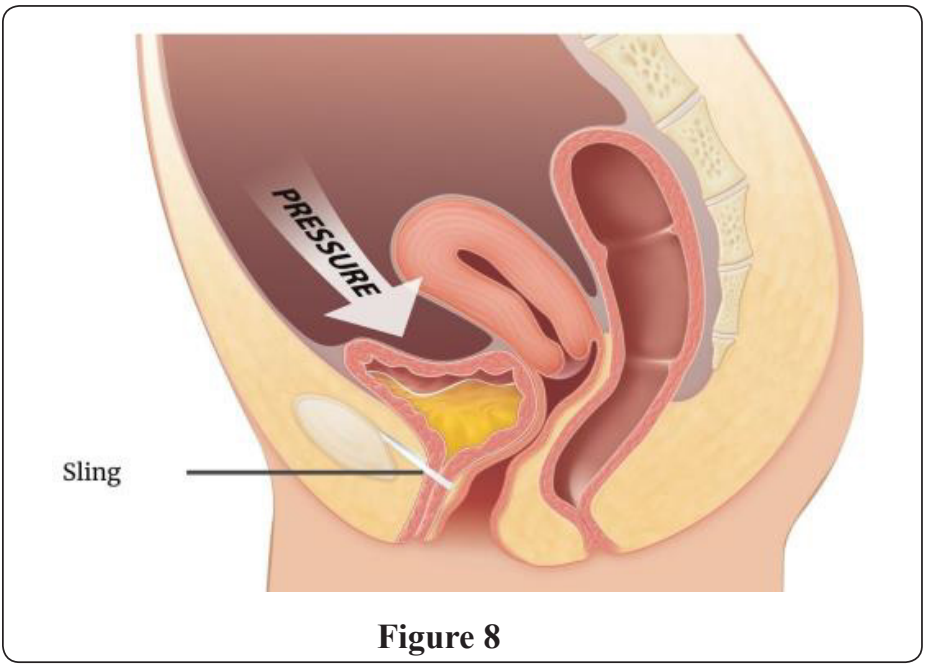

The surgical sling procedure's success rate is $80 \%$ at best, with a $30 \%$ risk of reoperation.

The leading causes of SUI include pregnancy, vaginal delivery, and pelvic floor muscles weaken, causing pelvic organs to prolapse. When the bladder prolapses (cystocele), it stretches, widen, and kinks the urethra, which leads to distortion of the urethral sphincters that become incompetent (Figure 9).

Therefore, anything that increases abdominal pressure, such as coughing, sneezing, bending over, lifting, or laughing, can pressure your bladder and cause urine leakage (Figure 10). Other factors that may worsen SUI include age, menopause, chronic coughing, obesity, jumping, and other factors. SUI causes emotional distress and embarrassment. It can disrupt work, social activities, relationships, and even sex life.
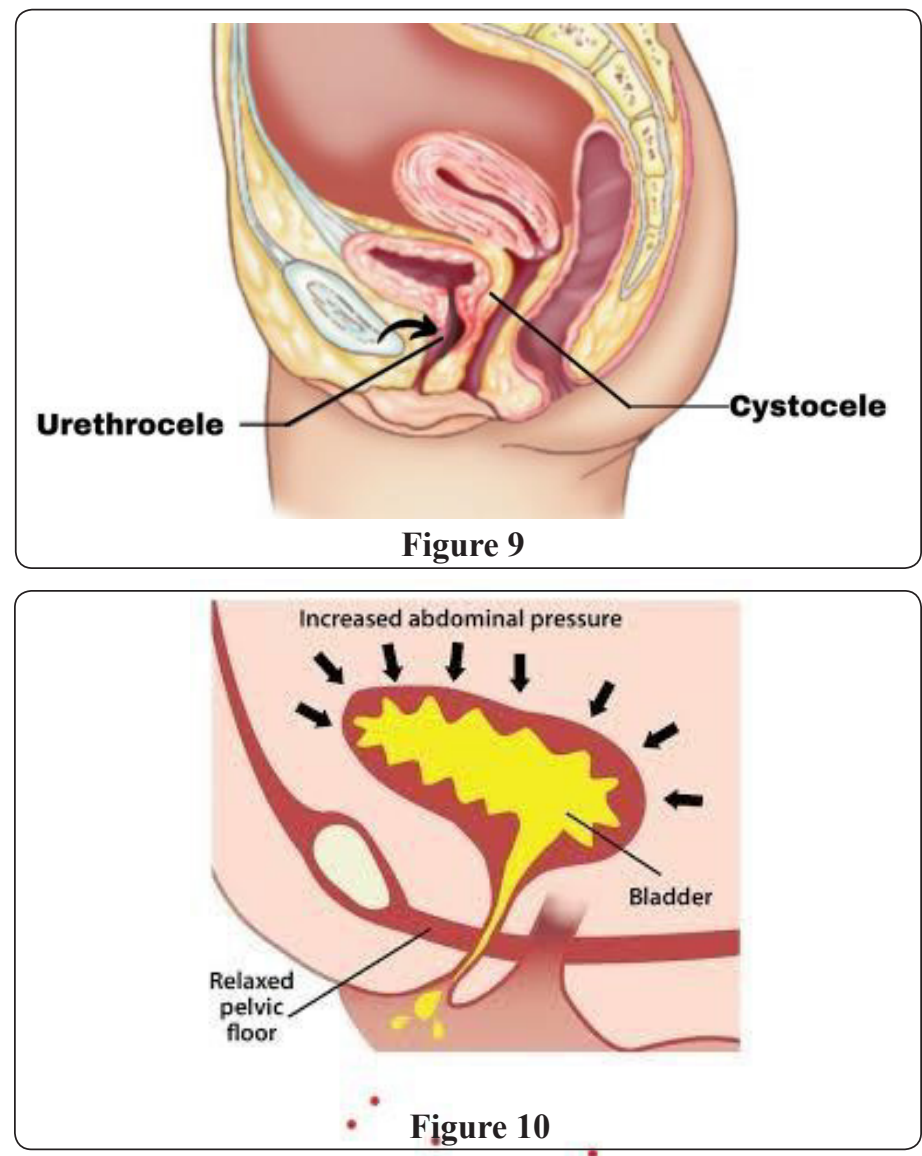

\section{Materials}

The FemCap combines the ring and space-occupying pessary features into one device, which makes it much more successful. Our investigation started by looking at the similarities between the FemCap and the ring pessary with support. The FemCap shows a marked resemblance to the ring pessary and the space-occupying 
pessary. The Rim of the FemCap is similar in shape and function to the ring pessary that supports the bladder neck. The brim's outward flaring pushes the cystocele forward, which restores the anatomy of the bladder and urethra where they belong (Figure 11). The bowl of the FemCap supports the cervix and prevents it from descending, which provides further support (Figure 3).

The FemCap has been in use for 20 years without any significant reportable side effects.

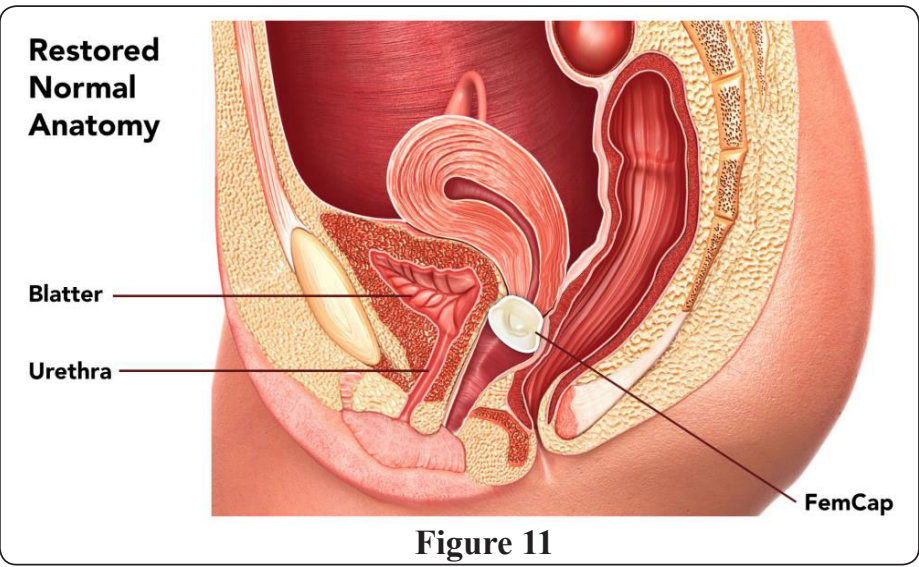

\section{Methods}

We recruited 41 women to be participants in a feasibility study for the management SUI. All the 41 women completed the protocol using the FemCap, to control their SUI for two weeks. We conducted an earlier pilot clinical trial to check the feasibility of the FemCap in controlling stress urinary incontinence [11].

\section{The Protocol has the Following}

\section{Inclusion Criteria}

a) women to be aged18 -75 old and

b) they must have at least two episodes of stress incontinence per day

c) they must be capable of inserting and removing the FemCap on their own, and

d) would be able to fill the Case Record Forms (CRF)

\section{Exclusion Criteria}

Women who had stage 3 or 4 prolapses, overactive bladder, overflow incontinence or pelvic infection or ulceration of the vagina

\section{Study Procedures \\ Enrollment Visit}

The investigator or his or her assistant explained the purpose of the study to the enrolee and how to fill the case record forms. If the enrolee is eligible for the study, the investigator performed a pelvic exam to rule out contraindications such as cancer, infection, or ulceration. If there is no reason for exclusion, the investigator showed her the FemCap and taught her how to insert and remove it. The investigator provided the CRF card and asked her to record any SUI episode and any side effects or problems.

\section{Second or Final Visit}

Interview the participant and checked the case records forms before using the FemCap (Figure 12) and after using the FemCap (Figure 13 ) to compare it to the previous CRF.

\section{Risk Analysis}

We did not anticipate any risk related to or from using the FemCap however; we ask all participants to report any issues they think may be related.

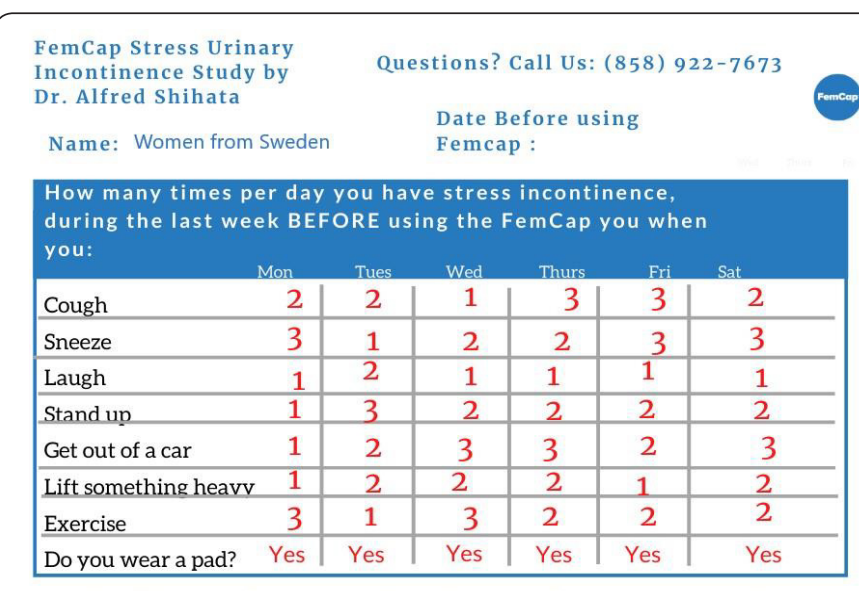

You should insert the FemCap first thing in the morning*. Don't remove it until bedtime and then wash and store it in container until you use it the next morning.

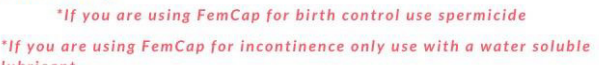

Figure 12

Date After using

FemCap :

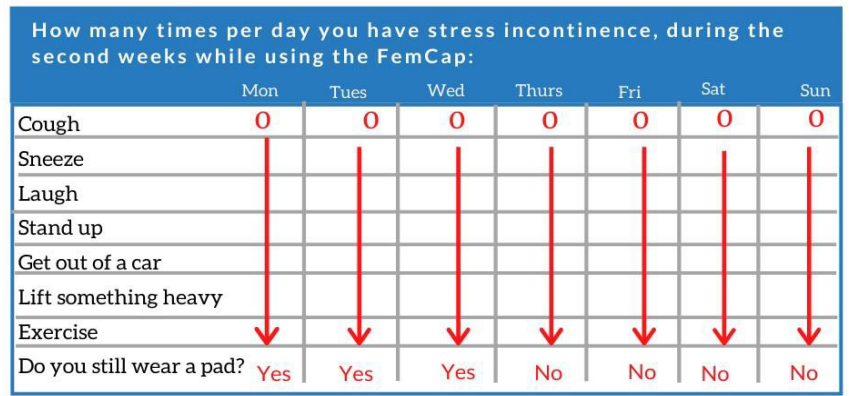

1. Did have any side effects from the FemCap? No

2. Will you consider using the FemCap in the Future to control your (SUI)? Yes

3. Comments Ididn't have leakage when jumping on the trampoline while using the FemCap, Coughing and sneezing no longer was an issue when I had a cold while using FemCap

Figure 13

\section{Results}

Thirty-four women out of 41 were completely dry after two weeks of using the FemCap, while four women were partially dry, and three women did not notice any change. The participants reported no side effects, and pelvic examinations did not show any erosion or ulceration of the vagina. 


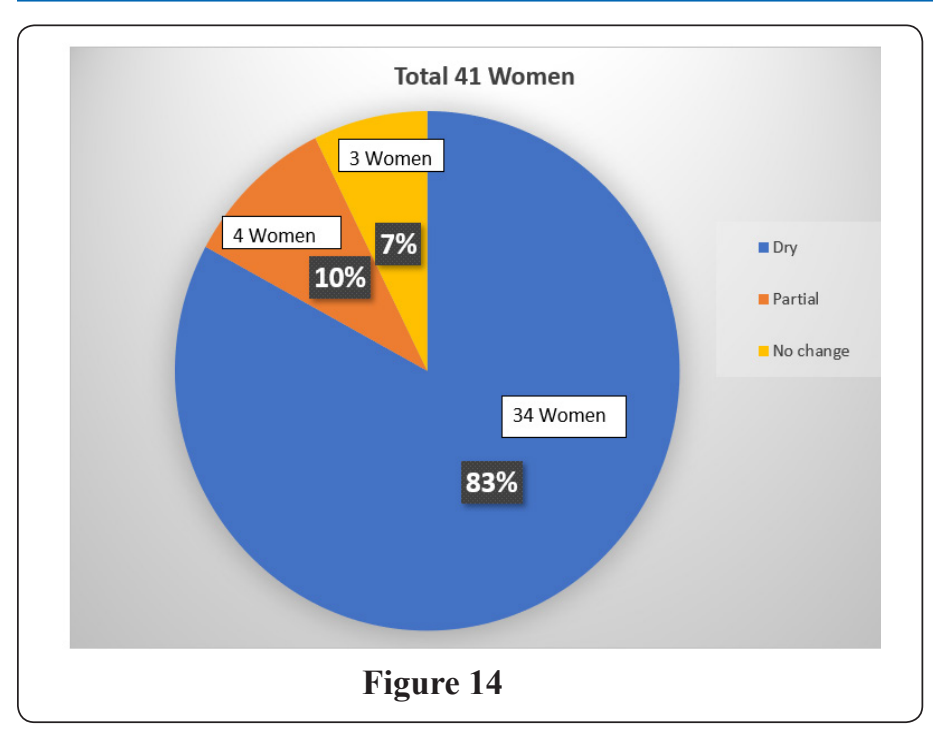

\section{Conclusion}

Currently, Stress urinary incontinence is an unspoken condition that women have to suffer silently with shame and embarrassment. The FemCap is safe and effective in restoring the bladder and urethra's anatomy, which could make it ideal for the treatment and prevention of mild to moderate SUI. More studies are warranted to prove the utility of the FemCap further to manage SUI [12-14].

\section{Reference}

1. Alfred A Shihata, James Trussell (1991) New Female Intravaginal Barrier Contraceptive Device Preliminary Clinical Trial, Contraception 44: 11-19.

2. Mauck C, Baker J, Barr S, W Johanson, DF Archer (1997) Phase I study of FemCap used with and without spermicide: Postcoital testing. Contraception 56: 111-115.

3. Mauck C, Callahan M, Weiner D, R Dominik (1999) A comparative study of the safety and efficacy of FemCap, a new vaginal barrier contraceptive, and the Ortho All-Flex ${ }^{\circledR}$ diaphragm. Contraception 60: 71-80.

4. Carcio H, Clarke Secor M, Koeniger Donohue R (2010) Advanced Health Assessment of Women: Clinical Skills and Procedures Chapter 15 The FemCap. Springer Publishing Company 2010, Pages 271-278.

5. Koeniger Donohue R (2006) The FemCap a Non-Hormonal Contraceptive, Women's Health Care NPWH 5: 79-91.

6. Shoupe D, Kjos S (2006) The Handbook of Contraception, Barrier Contraceptives Chapter 10 Humana Press 147-17.

7. Shihata A (1998) The FemCap ${ }^{\mathrm{TM}}$, a new contraceptive choice. Eur J Contracept and Reprod Health Care 3: 160-166.

8. Shihata A (2004) New FDA approved woman-controlled, latexfree barrier contraceptive device "FemCap ${ }^{\mathrm{TM}}$ " Fertil Steril. International Congress Series 1271 103: 303-306.

9. G Willy Davila (2009) Introl ${ }^{\mathrm{TM}}$ Bladder Neck Support Prosthesis: A Nonsurgical Urethropexy. Journal of Endourology 10: 293-296.

10. Danny Lovatsis, Mount Sinai Hospital, Canada A Randomized Controlled Trial of the Uresta Continence Pessary (SURE) ClinicalTrials.gov Identifier: NCT01284244 Recruitment Status: Completed First Posted: Jan 26, 2011 Results First Posted: Mar 31, 2017, Last Update Posted: Mar 31, 2017.

11. Shihata Alfred, Brody Steven (2020) Multipurpose, Reusable, Female Contraceptive Device That Enhances the Effectiveness of Fertility Awareness Methods and Controls Stress Incontinence. Medical Research Archives 8.

12. Viera AJ, Larkins-Pettigrew M (2000) Practical use of the pessary. Am Fam Physician 61: 2719-2729.

13. Al-Shaikh G, Syed S, Osman S, Bogis A, Al-Badr A (2018) Pessary use in stress urinary incontinence: a review of advantages, complications, patient satisfaction, and quality of life. Int J Women's Health 10: 195-201.

14. Jones K, Harmanli O (2010) Pessary Use in Pelvic Organ Prolapse and Urinary Incontinence. Reviews in Obstetrics \& Gynecology 3: 3-9.
Copyright: (C)2020 Shihata Alfred, et al. This is an open-access article distributed under the terms of the Creative Commons Attribution License, which permits unrestricted use, distribution, and reproduction in any medium, provided the original author and source are credited. 